\title{
Adrenal hypofunction associated with ashwagandha (Withania somnifera) supplementation: a case report
}

\author{
Christopher H. Fry ${ }^{1}$. David Fluck ${ }^{2}$ - Thang S. Han ${ }^{3,4}$ (I)
}

Accepted: 9 January 2022 / Published online: 14 February 2022

(c) The Author(s) 2022

\begin{abstract}
Objective The use of herbal medicinal supplements has gained huge popularity world-wide, but scientific evidence of their effectiveness and safety remains scarce. Ashwagandha (Withania somnifera) is one such product, claimed to alleviate pain and anxiety by lowering circulating cortisol levels. Withanolides, which are the principal bioactive compounds of ashwagandha, are naturally occurring steroids and may suppress adrenal function. Here, we describe the effect of ashwagandha on adrenal function of a 41-year-old woman with a low body mass index and who suffered chronic pain and lethargy.

Methods Adrenal function was assessed by the short Synacthen test (SST) during and after treatment with ashwagandha supplementation.

Results Whilst taking daily ashwagandha supplement (21.4 mg of Withanolides), for ten weeks, a SST showed a minimal response to $250 \mu \mathrm{g}$ of an intramuscular injection of Synacthen (tetracosactide): cortisol levels at $T_{0 \min }=287 \mathrm{nmol} / \mathrm{l}$, $T_{30 \min }=289 \mathrm{nmol} / 1$, and $T_{60 \min }=328 \mathrm{nmol} / 1$; from a morning baseline cortisol level of $480 \mathrm{nmol} / 1$ prior to taking the supplement. Ashwagandha was discontinued for two weeks, and a repeat SST was performed showing a completely normal adrenal response: cortisol level at $T_{0 \min }=275 \mathrm{nmol} / 1, T_{30 \min }=623 \mathrm{nmol} / 1$ and $T_{60 \min }=674 \mathrm{nmol} / 1$.

Conclusion Ten weeks of ashwagandha supplementation was associated with adrenal hypofunction, which was reversible after a two-week break. Individuals taking ashwagandha should be aware of this potentially detrimental effect. Future studies are suggested to assess whether long-term treatment with ashwagandha could lead to permanent suppression of adrenal function, and to elucidate the effects of ashwagandha compounds on adrenal steroidogenic pathway and hypothalamic-pituitary-adrenal axis.
\end{abstract}

Keywords Ayurveda $\cdot$ Dietary supplements $\cdot$ HPA axis $\cdot$ Hypocortisolism $\cdot$ Short Synacthen test $\cdot$ Steroidogenesis $\cdot$ Toxicity

Thang S. Han

thang.han@rhul.ac.uk

1 School of Physiology, Pharmacology and Neuroscience, University of Bristol, Bristol BS8 1TD, UK

2 Department of Cardiology, Ashford and St Peter's NHS Foundation Trust, Guildford Road, Chertsey KT16 0PZ, Surrey, UK

3 Department of Endocrinology, Ashford and St Peter's NHS Foundation Trust, Guildford Road, Chertsey KT16 0PZ, Surrey, UK

4 Institute of Cardiovascular Research, Royal Holloway, University of London, Egham TW20 0EX, Surrey, UK

\section{Introduction}

Over the past four decades, the use of herbal medicinal products and supplements has gained huge popularity worldwide. These products are sold on the Internet and in health shops, but many are not rigorously tested for their effectiveness and safety by clinical trials. Individuals with symptoms of generalised bodily pain and anxiety are particularly attracted to these supplements [1]. Ashwagandha (Withania somnifera) extract is one such herbal product marketed globally $[2,3]$. Although commonly known as ashwagandha, it has been called by some ten other names. An evergreen shrub, ashwagandha is a genus of flowering plants in the Solanaceae (nightshade) family, and most of it is grown in South Asia, the Middle East and North Africa, but may also be found in Southern Europe and the Mediterranean [4]. 
This plant has been used for thousands of years as a medicinal herb in traditional Ayurvedic medicine (Ayurveda). Withanolides, which are the principal bioactive compounds of ashwagandha, are naturally occurring steroids. About 35 steroidal withanolides, along with 12 steroidal alkaloids and several sitoindosides have been isolated from ashwagandha [5-7].

In modern days, ashwagandha is advertised widely for its apparent "multiple beneficial effects on a number of organs including the central nervous and endocrine systems, as well as the ability to alleviate pain by its anti-inflammatory property and generate calming effects by lowering adrenal steroids". However, similar to many other herbal medicinal products, adverse effects of ashwagandha on humans have not been well documented [2]. Here, we describe the effect of ashwagandha on the adrenal function of a woman.

\section{Results}

\section{Case presentation}

A 41-year-old woman was reviewed in the Endocrine clinic on 4 May 2021. She had a two-year history of generalised bodily pain sustained from a road traffic accident. To relieve pain she was treated with the tricyclic antidepressant amitriptyline at $25 \mathrm{mg}$ a day (amitriptyline has no known effects on the steroidogenic pathways), and she had never taken opioid-based drugs or steroids. She had been taking an oral contraceptive pill (OCP) but stopped one year previously. She had regular periods whilst not taking OCP. She had no history of weight loss and was always slim (body mass index $=17.5 \mathrm{~kg} / \mathrm{m}^{2}$ ). She did not smoke or drink alcohol excessively. Her thyroid function and basic haematological and biochemical parameters were normal; lyme borreliosis was also excluded (Table 1). A short Synacthen test (SST) to exclude adrenal insufficiency was suggested. Whilst SST was being arranged, the patient inadvertently visited the phlebotomy department where a morning random cortisol was done, showing a level of $480 \mathrm{nmol} / \mathrm{l}$. The formal SST was performed on 12 August 2021. To our surprise, the baseline cortisol level had dropped to $287 \mathrm{nmol} / \mathrm{l}$, whilst the response to $250 \mu \mathrm{g}$ of an intramuscular injection of Synacthen (tetracosactide) was minimal $\left(T_{30 \min }=289 \mathrm{nmol} / \mathrm{l}\right.$, and $\left.T_{60 \min }=328 \mathrm{nmol} / \mathrm{l}\right)$.

On direct questioning, the patient's symptoms remained unchanged. However, it became apparent that shortly after the initial consultation in May 2021, the patient began to conduct Internet search on health topics relating to the adrenal glands. She discovered a number of websites selling ashwagandha root extract, which was advertised for its "anti-inflammatory property and ability to lower cortisol levels, leading to reduction of pain and stress". The patient ordered this product (Clean
Table 1 Physiological and biochemical characteristics of the patient prior to taking ashwagandha supplement

\begin{tabular}{lll}
\hline Patient characteristics & Parameters & Reference range \\
\hline Age, years & 41 & - \\
Body mass index, $\mathrm{kg} / \mathrm{m}^{2}$ & $17.5^{*}$ & $20-25$ \\
$\begin{array}{l}\text { Systolic/diastolic blood pressure, } \\
\mathrm{mmHg}\end{array}$ & $120 / 75$ & $<160 / 90$ \\
Morning cortisol, nmol/1 & 480 & $>550^{\dagger}$ \\
Thyroid stimulating hormone, mU/l & 2.09 & $0.35-4.78$ \\
Haemoglobin, g/l & 124 & $115-165$ \\
Creatinine, $\mu \mathrm{mol} / \mathrm{l}$ & 60 & $<60$ \\
Alanine transferase, $\mathrm{U} / 1$ & 25 & $10-49$ \\
Calcium, mmol/l & 2.26 & $2.2-2.6$ \\
Ferritin, $\mu \mathrm{g} / \mathrm{l}$ & 169 & $15-250$ \\
Borrelia burgdorferi $\mathrm{IgG}$ & Not detected & - \\
\hline
\end{tabular}

${ }^{*}$ Weight $=50 \mathrm{~kg}$, height $=1.69 \mathrm{~m}$; ${ }^{\dagger}$ Some centres accept a random cortisol level of $>450 \mathrm{nmol} / \mathrm{l}$ as normal

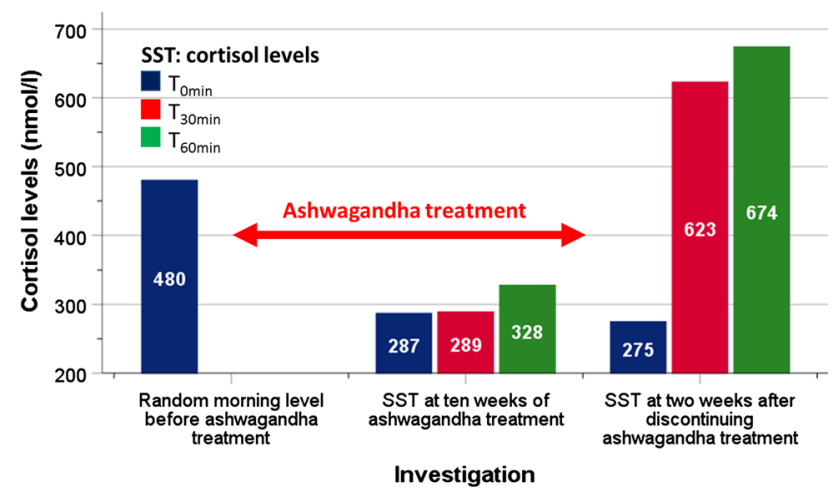

Fig. 1 Cortisol levels measured prior to, during treatment and after discontinuation of treatment with ashwagandha

Ashwagandha, British Supplements, UK) [8] and took one capsule twice a day up to the end of August 2021 (two capsules $=858.6 \mathrm{mg}$ ashwagandha extract, containing $21.4 \mathrm{mg}$ Withanolides, plus $95.4 \mathrm{mg}$ of the manufacturer's uptake blend). Therefore, the patient was taking ashwagandha for ten weeks by the time she had the first SST. Ashwagandha was discontinued for two weeks and a repeat SST was performed on 14 of September showing a completely normal adrenal response to Synacthen: cortisol level at $T_{0 \min }=275 \mathrm{nmol} / \mathrm{l}$, $T_{30 \min }=623 \mathrm{nmol} / 1$ and $T_{60 \min }=674 \mathrm{nmol} / 1$ (Fig. 1). The adrenocorticotropic hormone (ACTH) level at baseline $\left(T_{0 \min }\right)$ of this second SST was normal at $11 \mathrm{ng} / \mathrm{l}$ (reference range: $<50 \mathrm{ng} / \mathrm{l})$. 


\section{Discussion}

\section{Summary}

We present a case whose adrenal function was suppressed during the period when the patient was taking ashwagandha, which was reversed to normal function after discontinuation of this herbal product. As far as we are aware, this is the first observation of temporal changes in adrenal function, as assessed by SST, during and after stopping supplementation with ashwagandha. This case highlights the ability of ashwagandha to suppress adrenal function in a relatively short duration (ten weeks), but could be reversed after two weeks of discontinuation.

\section{Clinical implications}

There is a lack of scientific evidence of the effectiveness and safety of ashwagandha for treating any disease, and according to expert review from www.drugs.com website, trials supporting its clinical use in humans are limited [2]. Animal studies suggest it has effects on the immune, endocrine and central nervous systems, and inflammatory conditions. There are a handful of randomised controlled trials (RCT) from India $[9,10]$. A recent RCT of sixty healthy Indian adults showed supplementation with $240 \mathrm{mg}$ of ashwagandha extract once daily for 60 days led to a reduction in the Hamilton anxiety rating scale $(P=0.040)$ and morning cortisol levels $(P<0.001)$ compared with placebo [10]. The major flaws with such study were that there was no valid medical reason for lowering anxiety or cortisol levels of volunteers who were described as healthy adults. Cortisol reduction should indeed be interpreted as an adverse effect of ashwagandha rather than benefit. The subnormal adrenal response to tetracosactide (assessed by SST) observed in our patient could potentially lead to serious health consequences due to the inability of the patient to mount a response to an acute stress, such as a major illness or infection. As far as we are aware, there is no existing literature on dynamic endocrine tests of adrenal function (such as SST) during and after taking this supplement.

\section{Adverse effects}

A number of potential toxic actions associated with ashwagandha have been comprehensively reviewed and described by experts, including clinicians and pharmacists, on the www.drugs.com website [2]. Studies with Wistar rats showed that repeated injections of ashwagandha extract, at a dose of $100 \mathrm{mg} / \mathrm{kg}$ body weight for 30 days, led to reduction in the weights of adrenals, thymus and spleen [11], whilst hepatotoxicity effects of ashwagandha have recently been reported in humans $[12,13]$. Dosing in humans is variable, ranging from $120 \mathrm{mg}$ to $2 \mathrm{~g}$ a day. Contraindications and interactions and its use in pregnancy and lactation have not been well documented, but adverse effects are scarcely or inappropriately reported in human studies. For example, in two recent RCTs, one reported "no adverse events" [9] whilst the other inadequately monitored treatment safety by full blood counts and lipids [10]. Since there is no existing published literature on adrenal function amongst people taking ashwagandha, it is not possible to determine if this herbal product affects the adrenal function differently in people of different age, sex, body composition or ethnic background.

\section{Mechanisms}

Plants have evolved to produce a number of toxic substances as defence mechanisms against predation from microorganisms (bacteria and fungi), insects and animals [14-17]. A large number of plants, including ashwagandha, used in herbal medicines possess this toxic property. The withanolides from ashwagandha contain a highly oxygenated $\mathrm{C} 28$ ergostane-type steroidal nucleus with C22-hydroxy-C26-oic acid $\delta$-lactone in a nine-carbon side chain, and oxidised to form a six-membered lactone ring [18-20]. It is possible that the steroidal compounds from ashwagandha, such as withanolides and alkaloids, may have a direct effect on adrenal function. Adrenal steroidogenesis is complex, involving a pathway of precursor hormones that require specific enzymatic steps [21], the genes that encode the enzymes involved in the control of steroid biosynthesis may be interrupted by withanolides and alkaloids, giving rise to adrenal hypofunction. A recent study has shown that withanone, a bioactive constituent of withanolides found in ashwagandha extract, may cause deoxyribonucleic acid (DNA) damage by forming adducts to DNA. Withanone also forms adducts with amines, which are reversibly detoxified by glutathione (GSH) but may cause DNA damage when the GSH system is overwhelmed by excessive levels of withanone [22]. Studies have also found that alkaloids from a number of herbal medicines react with DNA, causing cellular toxicity or genotoxicity (damage to the genome), leading to structural alterations of the genetic material through induction of DNA binding and cross-linking, as well as chromosomal abnormalities [23].

The effects of steroidal compounds from ashwagandha may extend to higher neuroendocrine centres controlled by the hypothalamus and pituitary. The hypothalamic-pituitary axis is known to be vulnerable to stress from restricted dietary practice and excessive exercise [24] and a number of drugs including exogenous steroids and opioids [25]. It is plausible that the steroidal withanolides and alkaloids 
from ashwagandha could suppress the hypothalamic-pituitary-adrenal (HPA) axis, in a similar way that exogenous corticosteroids (used to treat chronic inflammatory conditions) do to the HPA axis, leading to hypoadrenalism [25, 26].

\section{Patient perspective}

The patient expressed that she would take greater caution before considering taking any dietary supplements in the future. She would do thorough research on independent sources and consult with healthcare professionals.

\section{Materials and methods}

Information for demographic factors and medications and physiological measurements were obtained from clinical history and examination. Blood was taken for biochemistry and haematology investigations. Adrenal function was assessed by SST: the levels of cortisol were obtained at baseline (prior to Synacthen injection), and at $30 \mathrm{~min}$ and $60 \mathrm{~min}$ after an intramuscular injection of $250 \mu \mathrm{g}$ of Synacthen into the deltoid muscle. An incremental rise of cortisol level by at least $200 \mathrm{nmol} / 1$ or a peak cortisol of $>550 \mathrm{nmol} / \mathrm{l}$ was considered as a normal adrenal response. The SST was performed whilst the patient was taking ashwagandha and two weeks after coming off this supplement. Analyses were performed using SPSS Statistics for Windows, v.25.0 (IBM Corp., Armonk, NY, USA).

\section{Conclusion}

This study shows that a relatively short course of ashwagandha is associated with adrenal hypofunction, which was reversible after two weeks of discontinuation of supplementation. Individuals taking ashwagandha should be aware of its detrimental effects. Future studies are suggested to assess whether long-term treatment with ashwagandha could lead to permanent suppression of adrenal function. Further in vitro and in vivo studies are necessary to elucidate the effects of ashwagandha compounds on adrenal steroidogenic pathway and HPA axis.

Acknowledgements We are thankful to our patient for her thorough discussion of her condition and to consent that her case be published in a peer-reviewed scientific medical journal. We are also grateful for additional information on ashwagandha provided by Master Alasdair KF Han (St Christopher's Preparatory School, Middlesex), and
Professor Michael EJ Lean (Department of Human Nutrition, University of Glasgow) for his insightful comments.

\section{Declarations}

Conflict of interest Christopher H. Fry, David Fluck and Thang S. Han declare that they have no conflicts of interest.

Ethical approval This study does not require NHS Research Ethics Committee approval since it involves secondary analysis of anonymised data. This study was conducted in accordance with the 1964 Helsinki declaration and its later amendments or comparable ethical standards.

Statement of human and animal rights This article does not contain any studies with animals performed by any of the authors.

Statement of authorship TSH reviewed the topic related literature and wrote the first draft, interpreted the data and revised the manuscript. $\mathrm{CHF}$ and DF checked, interpreted results and commented on the manuscript. All authors critically revised the manuscript and agree to be fully accountable for ensuring the integrity and accuracy of the work and read and approved the final manuscript.

Open Access This article is licensed under a Creative Commons Attribution 4.0 International License, which permits use, sharing, adaptation, distribution and reproduction in any medium or format, as long as you give appropriate credit to the original author(s) and the source, provide a link to the Creative Commons licence, and indicate if changes were made. The images or other third party material in this article are included in the article's Creative Commons licence, unless indicated otherwise in a credit line to the material. If material is not included in the article's Creative Commons licence and your intended use is not permitted by statutory regulation or exceeds the permitted use, you will need to obtain permission directly from the copyright holder. To view a copy of this licence, visit http://creativecommons.org/licenses/by/4.0/.

\section{References}

1. Ekor M (2014) The growing use of herbal medicines: issues relating to adverse reactions and challenges in monitoring safety. Front Pharmacol 4:177. https://doi.org/10.3389/fphar.2013.00177

2. XXX. https://www.drugs.com/npp/ashwagandha.html. Accessed on 200 Sept 2021

3. Bodeker G, Ong CK (2005) WHO global atlas of traditional, complementary and alternative medicine. World Health Organization, New York

4. Germplasm Resources Information Network (GRIN). Agricultural Research Service (ARS), United States Department of Agriculture (USDA). https://npgsweb.ars-grin.gov/gringlobal/taxon/taxon omydetail?id=102407. Accessed on 20 Sept 2021

5. Kulkarni SK, Dhir A (2008) Withania somnifera: an Indian ginseng. Prog Neuropsychopharmacol Biol Psych 32:1093-1105. https://doi.org/10.1016/j.pnpbp.2007.09.011

6. Mishra LC, Singh BB, Dagenais S (2000) Scientific basis for the therapeutic use of Withania somnifera (ashwagandha): a review. Altern Med Rev 5:334-346

7. Matsuda H, Murakami T, Kishi A, Yoshikawa M (2001) Structures of withanosides I, II, III, IV, V, VI, and VII, new withanolide glycosides, from the roots of Indian Withania somnifera DUNAL and inhibitory activity for tachyphylaxis to clonidine in isolated 
guinea-pig ileum. Bioorg Med Chem 9:1499-1507. https://doi. org/10.1016/s0968-0896(01)00024-4

8. XXX. https://www.british-supplements.net/products/clean-genui ne-ashwagandha-extract-veg-caps-9-020mg-22-54mg-of-witha ndides. Accessed on 20 Sept 2021

9. Langade D, Kanchi S, Salve J, Debnath K, Ambegaokar D (2019) Efficacy and safety of Ashwagandha (Withania somnifera) root extract in insomnia and anxiety: a double-blind, randomized, placebo-controlled study. Cureus 28(11):e5797. https://doi.org/ $10.1155 / 2015 / 284154$

10. Lopresti AL, Smith SJ, Malvi H, Kodgule R (2019) An investigation into the stress-relieving and pharmacological actions of an ashwagandha (Withania somnifera) extract: a randomized, doubleblind, placebo-controlled study. Medicine 98:e17186. https://doi. org/10.1097/MD.0000000000017186

11. Sharada AC, Solomon FE, Devi PU (1993) Toxicity of Withania somnifera root extract in rats and mice. Int J Pharmac 31:205-212. https://doi.org/10.3109/13880209309082943

12. Björnsson HK, Björnsson ES, Avula B, Khan IA, Jonasson JG, Ghabril M, Hayashi PH, Navarro V (2020) Ashwagandha-induced liver injury: a case series from Iceland and the US drug-induced liver injury network. Liver Int 40:825-829. https://doi.org/10. 1111/liv.14393

13. Philips CA, Ahamed R, Rajesh S, George T, Mohanan M, Augustine $\mathrm{P}$ (2020) Comprehensive review of hepatotoxicity associated with traditional Indian Ayurvedic herbs. World J Hepatol 12:574595. https://doi.org/10.4254/wjh.v12.i9.574

14. Selitrennikoff CP (2001) Antifungal proteins. Appl Environ Microbiol 67:2883-2894. https://doi.org/10.1128/AEM.67.7. 2883-2894.2001

15. Rates SMK (2001) Plants as source of drugs. Toxicon 39:603613. https://doi.org/10.1016/s0041-0101(00)00154-9

16. Vernekar JV, Ghatge MS, Deshpande VV (1999) Alkaline protease inhibitor: a novel class of antifungal proteins against phytopathogenic fungi. Biochem Biophys Res Commun 262:702-707. https://doi.org/10.1006/bbrc.1999.1269

17. Priyandoko D, Ishii T, Kaul SC, Wadhwa R (2011) Ashwagandha leaf derived withanone protects normal human cells against the toxicity of methoxyacetic acid, a major industrial metabolite. PLoS ONE 6:e19552. https://doi.org/10.1371/journal.pone.00195 52

18. Zhang H, Timmermann BN (2016) Withanolide structural revisions by $13 \mathrm{C}$ NMR spectroscopic analysis inclusive of the $\gamma$-gauche effect. J Nat Prod 79:732-742. https://doi.org/10.1021/ acs.jnatprod.5b00648

19. Vaishnavi K, Saxena N, Shah N, Singh R, Manjunath K, Uthayakumar M, Kanaujia SP, Kaul SC, Sekar K, Wadhwa R (2012) Differential activities of the two closely related withanolides, Withaferin A and Withanone: bioinformatics and experimental evidences. PLoS ONE 7:e44419. https://doi.org/10.1371/journal. pone.0044419

20. Mirjalili MH, Moyano E, Bonfill M, Cusido RM, Palazón J (2009) Steroidal lactones from Withania somnifera, an ancient plant for novel medicine. Molecules 14:2373-2393. https://doi.org/10. 3390/molecules 14072373

21. Han TS, Walker BR, Arlt W, Ross RJ (2014) Treatment and health outcomes in adults with congenital adrenal hyperplasia. Nat Rev Endocrinol 10:115-124. https://doi.org/10.1038/nrendo.2013.239

22. Siddiqui S, Ahmed N, Goswami M, Chakrabarty A, Chowdhury G (2004) DNA damage by Withanone as a potential cause of liver toxicity observed for herbal products of Withania somnifera (Ashwagandha). Drug Metab Rev 36:1-55. https://doi.org/10.1081/ dmr-120028426

23. Fu PP, Xia Q, Lin G, Chou MW (2004) Pyrrolizidine alkaloidsgenotoxicity, metabolism enzymes, metabolic activation, and mechanisms. Drug Metab Rev 36:1-55. https://doi.org/10.1081/ dmr-120028426

24. Nyekiova M, Ghaderi S, Han TS (2014) Carotenoderma in a young woman of normal body mass index with hypothalamic amenorrhoea: a 2-year follow-up case report. Eur J Clin Nutr 68:1362-1364. https://doi.org/10.1038/ejcn.2014.128

25. Bornstein SR, Bornstein TD, Andoniadou CL (2019) Novel medications inducing adrenal insufficiency. Nat Rev Endocrinol 15:561-562. https://doi.org/10.1038/s41574-019-0248-9

26. Prete A, Bancos I (2021) Glucocorticoid induced adrenal insufficiency. BMJ 374:n1380. https://doi.org/10.1136/bmj.n1380 